Volume 2

Number 2 Teaching Secrecy

January 2021

\title{
Secrecy in U.S. National Security: Why a Paradigm Shift Is Needed
}

Steven Aftergood

Project on Government Secrecy, Federation of American Scientists, saftergood@fas.org

Follow this and additional works at: https://scholarworks.sjsu.edu/secrecyandsociety

Part of the American Politics Commons, and the Public Affairs, Public Policy and Public Administration Commons

\section{Recommended Citation}

Aftergood, Steven. 2021. "Secrecy in U.S. National Security: Why a Paradigm Shift Is Needed." Secrecy and Society 2(2). https://doi.org/10.31979/2377-6188.2021.020210 https://scholarworks.sjsu.edu/secrecyandsociety/vol2/iss2/10

This Book Review is brought to you for free and open access by the School of Information at SJSU ScholarWorks. It has been accepted for inclusion in Secrecy and Society by an authorized administrator of SJSU ScholarWorks. For more information, please contact scholarworks@sjsu.edu.

\section{(c) (1)}

This work is licensed under a Creative Commons Attribution 4.0 License. 


\section{Secrecy in U.S. National Security: Why a Paradigm Shift Is}

Needed

\section{Keywords}

classification, Thomas S. Kuhn, national security, paradigm, secrecy 
Review, Secrecy in U.S. National Security: Why a Paradigm Shift Is Needed by James B. Bruce, Sina Beaghley, and W. George Jameson

Reviewed by Steven Aftergood ${ }^{1}$

Secrecy in U.S. National Security: Why a Paradigm Shift Is Needed, Santa Monica, CA, RAND Corp, 2018. 35 pp. / https://doi.org/10.7249/PE305

The national security classification system today is in an advanced state of decay. It neither protects secrets reliably against unauthorized disclosure and espionage nor releases them to the public when their sensitivity has diminished or lapsed. So it is well past time to remedy this situation, a RAND Corporation study concluded in 2018. And more than simple fixes will be needed. Bruce, Beaghley, and Jameson (n.d.), the authors of the RAND study, suggest that "to achieve meaningful improvements in secrecy reform, tinkering at the margins must yield to systemic changes." The authors go on to observe that a

A much-improved system will afford significantly better protection to secrets that truly need it; reduce complexity, subjectivity, and overclassification by providing clear parameters for creating secrets; and more fully support government transparency goals. (Bruce, Beaghley, and Jameson n.d.)

According to the authors, the many previous efforts to address the problem of secrecy in the research literature were hampered "by the 1 Steven Aftergood (@saftergood) directs the Project on Government Secrecy at the Federation of American Scientists, https://fas.org/sgp/ 
absence of any conceptual framework with the theoretical power and reach needed to address the modernization of secrecy" (Bruce, Beaghley, and Jameson 2018, 3).

The study's claim to novelty is therefore the use of the "paradigm concept" advanced by Thomas S. Kuhn - which refers to the structure, culture, rules and technologies of secrecy as well as the processes by which they are employed - to help analyze the failures of secrecy and to identify needed reforms (Bruce, Beaghley, and Jameson 2018, 2-3):

Our use of the paradigm concept presents a basis for comprehensive theoretical insights into secrecy [along with] ideas and hypotheses to create evidence-based policy recommendations that can mitigate or reverse secrecy performance failings. (3)

Those with an interest in national security secrecy can read the report, which is not very long, and decide for themselves how productive the new analytical framework is and how actionable the ensuing recommendations are likely to be. In my view, the results are underwhelming. Beyond reiterating the need for change, the study provides little practical guidance for transforming the status quo. Few if any of the recommendations are altogether new. Some are well-worn (e.g., enforce the "need-to-know" principle); others were proposed decades ago to no effect (e.g., legislate a comprehensive secrecy and transparency statute); and yet others are already underway to some degree (e.g., reduce the number of cleared personnel). 
The authors, who are national security professionals with deep experience in classification matters, are certainly correct that secrecy policy would benefit from greater theoretical insight and analytical rigor. But that worthy objective is not fully realized in this study, which is best understood as part of an ongoing conversation about secrecy, and not as a final conclusion or a finished product.

In the spirit of continued conversation prompted by the RAND study, the following points might be considered for further discussion:

- What is a secret? The study defines a secret as "any" national security information that has been classified by the government. But this overlooks important qualitative differences that exist among different categories of secrecy. A confidential diplomatic communication poses different security challenges than the secret design of a submarine navigation system, for example, and both are different from the details of a pending hostage rescue mission. They are not interchangeable in terms of the type of damage they could cause, or the duration of their sensitivity, or the degree of public interest in their disclosure. To call them all "secrets" and to then advocate greater centralization in their handling as this study does might be a mistake. Multiple tailored systems of information security could make more sense than a single policy of enforced uniformity. 
- "Overclassification" by itself is not a meaningful complaint. Like nearly every critic and commission concerned with secrecy policy, the current study broadly condemns "overclassification." But doing so does not advance the discussion because of the simple fact that there is no one who is in favor of overclassification! To gain traction on real secrecy issues, a more penetrating critique - or at least greater specificity - is needed. If one identifies a particular item of information that one believes is overclassified, there will almost always be someone who will defend its classification as appropriate (except in cases of clear error). Frequent disagreements over the legitimacy of specific instances of secrecy point to the need for better mechanisms for adjudicating such disputes. Currently it is mostly up to the originating agency - which classified the information in the first place - to decide whether or not to sustain its own position. A more disinterested decision making process would likely do a better job of both credibly protecting legitimate secrets and compelling disclosure of information that should be made public. This would be a structural change with a potentially high payoff.

- Personnel might be the missing link. The RAND report is silent on the people are who make and implement secrecy policy. But the character 
and the conduct of the classifiers might be a big part of the problem confronting the secrecy system, or a big part of the solution. Even cursory experience with official secrecy indicates that not all government officials are cut from the same cloth or behave in the same way. Some have a principled commitment to constitutional government, open society, and public accountability. Others are understandably more concerned with accomplishing their assigned mission efficiently and with a minimum of friction. The former will more willingly accept the complications that can result from increased transparency. If that is the goal, the policy question then becomes, how can such persons be identified, encouraged and rewarded?

- The secrecy paradigm is already changing due to external factors. If we had to wait for a new consensus in favor of the RAND report's various recommendations on secrecy reform, we would likely be waiting for a long time. But instead, for better or worse, the reality and the scope of official secrecy are changing before our eyes. Perhaps the most dramatic example is the case of commercial satellite imagery, which is already superior in many respects to the most highly classified intelligence satellite imagery of the late cold war era. Yet it is publicly available for a reasonable fee, and it has already made the world more transparent in a qualitatively new way. Similar developments are 
evident in high-speed computing, encryption, open source intelligence, and many other areas. And while only a small fraction of the US population is to be found on the front lines of traditional military conflicts, we are all on the front lines of offensive cyber activities, foreign information operations, climate change and other new types of national security threats. In such areas, we all have a "need to know" that demands satisfaction.

In short, there are many fundamental issues in national security classification policy that remain to be addressed, along with many opportunities for improved performance that remain to be seized.

\section{References}

Bruce, James B., Sina Beaghley, and W. George Jameson. n.d. Secrecy in U.S. National Security Why a Paradigm Shift Is Needed. RAND Corporation. https://www.rand.org/pubs/perspectives/PE305.html 